\title{
Direct 213 nm UV written Bragg gratings and waveguides in planar silica without hydrogen loading
}

\author{
Q.S. Ahmed, P.C. Gow, P.L. Mennea, R.H.S. Bannerman, D.H. Smith, C. Holmes, \\ J.C. Gates, P.G.R. Smith \\ Optoelectronics Research Centre, University of Southampton, Southampton, SO17 1BJ, UK \\ qazi.salman.ahmed@soton.ac.uk
}

\begin{abstract}
We report the first demonstration of integrated waveguides and Bragg gratings direct UV written into planar silica using a fifth harmonic $213 \mathrm{~nm}$ laser without hydrogen loading. We observe $0.44 \pm 0.26 \mathrm{~dB} \mathrm{~cm}^{-1}$ propagation loss. () 2020 The Author(s)
\end{abstract}

\section{Introduction}

Direct UV Writing (DUW) is an established technique for definning low-loss channel waveguides, Bragg gratings, directional couplers, and polarisers in photosensitive planar silica. The technique relies on photosensitivity of the core layer which is achieved by doping silica with germanium and boron. DUW uses a continuous-wave frequency-doubled argon-ion laser beam operating at $244 \mathrm{~nm}$ wavelength and average power up to $100 \mathrm{~mW}$ [1]. The doped silica layers require hydrogen loading to ensure sufficient refractive index change during $244 \mathrm{~nm}$ UV writing. Hydrogen loading is a time consuming process, which requires leaving the samples at high pressures $(\approx 120$ bar $)$ in a hydrogen cell for several days. Furthermore, outgassing of hydrogen during writing results in a time-dependent variation of final written structures and makes it more challenging to define more complex planar circuitry. Recently, fifth harmonic solid-state $\mathrm{Nd}: \mathrm{YVO}_{4}$ lasers operating at $213 \mathrm{~nm}$ wavelength have become commercially available, which provide nanosecond pulses with a high peak power. Compared with a typical argonion laser this source can be compact and cost-effective in terms of footprint and power consumption. In the past this new laser source has been used to produce strong Bragg gratings within the core of hydrogen-free SMF-28 fibre using a phase mask technique [2] and single-mode waveguides in hydrogen-loaded planar silica [3].

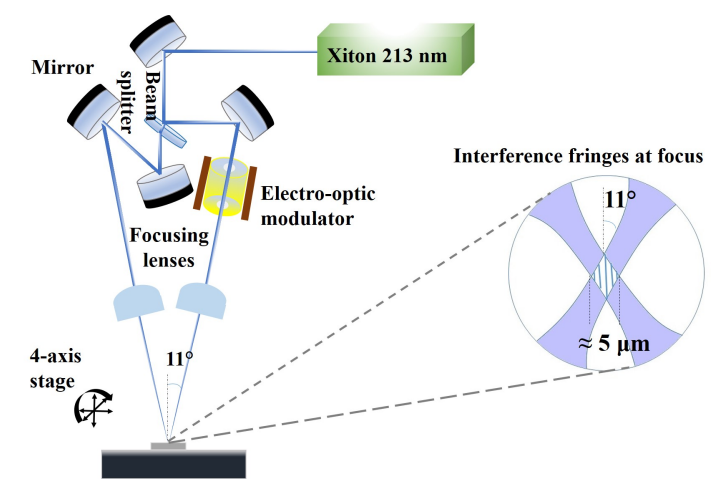

Fig. 1. Schematic of the $213 \mathrm{~nm}$ direct UV writing setup for defining channel waveguides and Bragg gratings simultaneously in planar silica layers.

Here we report the first successful fabrication of integrated waveguides and Bragg gratings by $213 \mathrm{~nm}$ direct UV writing in both hydrogen loaded and hydrogen-free planar silica samples. We characterise various properties of the waveguides including fluence dependence and propagation loss [4].

\section{Method}

Flame Hydrolysis Deposition (FHD) was used to deposit a $4.3 \mu \mathrm{m}$ thick core layer of germanium and boron doped-silica onto a silicon wafer with a $15 \mu \mathrm{m}$ thick thermal oxide. The core layer was capped with boron and phosphorus-doped $15 \mu \mathrm{m}$ thick silica cladding layer. The core and cladding layers were fabricated to have similiar refractive indices. $20 \mathrm{~mm}$ long individual chips were diced from this wafer, and several were hydrogen loaded 
in a pressure cell for several days. A $213 \mathrm{~nm}$ laser was used to simultaneously write waveguides and Bragg gratings within the photosensitive core layer through the small spot direct UV grating writing technique [1] as shown in Fig. 1. An average power of $12 \mathrm{~mW}$ and a Gaussian spot size of $5 \mu \mathrm{m}$, determined through knife edge measurements was used to write the hydrogen-loaded chip. Writing fluence for each waveguide was controlled by the stage translation speed, and a fluence range of $0.5 \mathrm{~kJ} \mathrm{~cm}^{-2}$ to $1.1 \mathrm{~kJ} \mathrm{~cm}^{-2}$ was used. Each written waveguide contained ten $1 \mathrm{~mm}$-long Bragg gratings with a Gaussian apodisation profile. The periods of gratings were changed to produce a spectral response between 1520 and $1590 \mathrm{~nm}$. The hydrogen-free sample was simialary inscribed with waveguides integrated with ten $1.5 \mathrm{~mm}$-long gratings with a fluence range of $20 \mathrm{~kJ} \mathrm{~cm}^{-2}$ to $100 \mathrm{~kJ} \mathrm{~cm}^{-2}$. Higher fluences were required to achieve a sufficient change in the refractive index. In order to avoid the damage threshold of FHD glass, the average power of the laser was reduced to $4 \mathrm{~mW}$. The total writing time for five waveguides was about 17 hours. For characterisation, an Er-doped ASE broadband source was launched into the UV written waveguides through a fibre $\mathrm{V}$-groove assembly. The reflected grating spectra were collected on an optical spectrum analyser.

\section{Results}

Fig. 2(a) shows a photograph of hydrogen-free fabricated chip. It shows the diffraction of the white light illumination source from the Gaussian apodised gratings. Fig. 2(b) shows an example reflection spectra observed from opposite ends of the waveguide. Maximum peak reflectivity of the gratings was observed $(\approx 20 \%)$ at a fluence of $60 \mathrm{~kJ} \mathrm{~cm}^{-2}$. Propagation loss was obtained using the Bragg grating reflectivity technique [4]. The measured loss was $0.44 \pm 0.26 \mathrm{~dB} \mathrm{~cm}^{-1}$ for $213 \mathrm{~nm}$ written hydrogen-free silica and $0.28 \pm 0.13 \mathrm{~dB} \mathrm{~cm}^{-1}$ in hydrogen loaded silica.

(a)

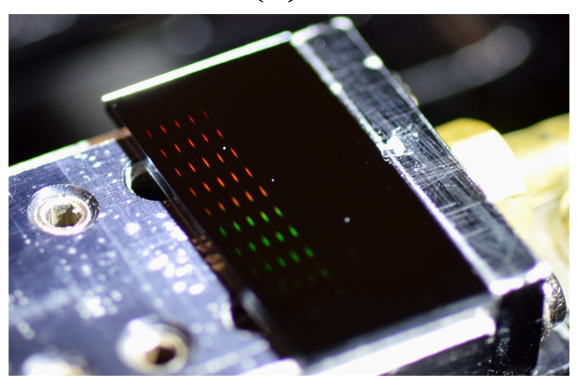

(b)

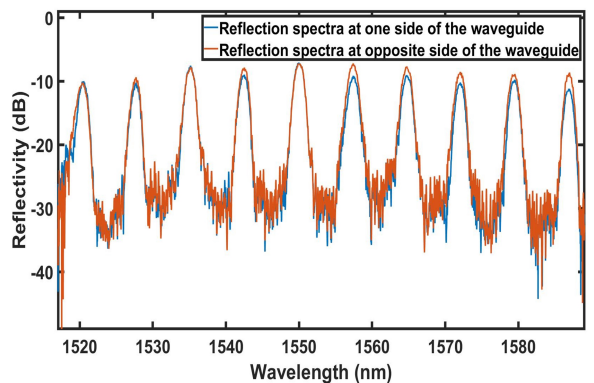

Fig. 2. (a) Photograph of a fabricated chip taken during characterisation, which shows a colorful scattering of white light from the Gaussian apodised gratings.(b) Reflection spectra obtained from launching lights from both end facets of the chip.

\section{Conclusion}

We have shown for the first time the successful fabrication of integrated waveguides and Bragg gratings in silicaon-silicon chips (with and without hydrogen loading) by $213 \mathrm{~nm}$ DUV writing. A Bragg grating based technique was utilised to measure the propagation loss of $213 \mathrm{~nm}$ written waveguides. The lowest propagation loss observed was $0.44 \pm 0.26 \mathrm{~dB} \mathrm{~cm}^{-1}$ in hydrogen free and $0.28 \pm 0.13 \mathrm{~dB} \mathrm{~cm}^{-1}$ in hydrogen loaded silica. We will present an in-depth comparison between hydrogen loaded and hydrogen free chips written with $213 \mathrm{~nm}$ laser, including the trend in propagation loss as a function of fluence, birefringence measurements and modal properties.

\section{References}

1. C. Sima, J. C. Gates, H. L. Rogers, P. L. Mennea, C. Holmes, M. N. Zervas, and P. G. R. Smith, "Ultra-wide detuning planar Bragg grating fabrication technique based on direct UV grating writing with electro-optic phase modulation," Opt. Express 21, 15747-15754 (2013).

2. M. Gagné and R. Kashyap, "New nanosecond Q-switched Nd: $\mathrm{VO}_{4}$ laser fifth harmonic for fast hydrogenfree Fiber Bragg Gratings fabrication,” Opt. Commun. 283, 5028-5032 (2010).

3. P. C. Gow, R. H. S. Bannerman, P. L. Mennea, C. Holmes, J. C. Gates, and P. G. R. Smith, "Direct UV written integrated planar waveguides using a 213 nm laser," Opt. Express 27, 29133-29138 (2019).

4. H. L. Rogers, S. Ambran, C. Holmes, P. G. R. Smith, and J. C. Gates, "In situ loss measurement of direct UV-written waveguides using integrated Bragg gratings,” Opt. Lett. 35, 2849-2851 (2010). 